\title{
Waveguide Immunofluorescence Sensor for Water Pollution Analysis
}

\author{
R.D. Harris ${ }^{a}$, G.R. Quigley ${ }^{a}$, J.S. Wilkinson ${ }^{a}$, A. Klotz ${ }^{b}$, C. Barzen ${ }^{b}$, A. Brecht ${ }^{b}$, G. Gauglitz ${ }^{b}$ and R.A. \\ Abuknesha ${ }^{c}$ \\ aptoelectronics Research Centre, Southampton University, UK \\ ${ }^{b}$ Institut für Physikalische und Theoretische Chemie, Eberhard-Karls-Universität, Tübingen, Germany \\ ${ }^{\mathrm{c}}$ GEC-Marconi Materials Technology Ltd., Borehamwood, UK
}

\begin{abstract}
A regenerable channel waveguide fluorescence sensor for environmental monitoring is reported. The sensor has been characterised as a detector of the pesticide 2,4 dichlorophenoxyacetic acid. A binding inhibition assay, using fluorescent Cy5.5 dye-labeled antibodies, was monitored at the modified surface of the glass waveguide to detect the target analyte. Three calibration curves were determined and averaged. The averaged calibration curve has a mid-point of $0.68 \mathrm{ppb}$ and a calculated detection limit of $0.28 \mathrm{ppb}$. Incorporation of a $20 \mathrm{~nm}$ thick tantalum pentoxide film at the waveguide surface enhanced the peak fluorescence signal by a factor of approximately 6 compared with an uncoated sensor.

Due to the high optical field strengths at the surface of the waveguide, which is approximately $10 \mu \mathrm{m}$ wide, significant photobleaching of the dye molecules occurs. The rate of photobleaching will be reduced if the power density of the excitation radiation at the surface of the waveguide is reduced, offering the potential for enhanced device sensitivity. It is demonstrated that this may be achieved, without reducing the total power, by broadening the $10 \mu \mathrm{m}$ wide optical waveguide through a tapered region to a final width in excess of $50 \mu \mathrm{m}$. A distinct advantage of this broadening is to improve the signal to noise ratio of the sensor as the number of bound fluorophores at the waveguide surface increases linearly with the waveguide width. Theoretical modeling of tapered waveguides, using a commercial beam propagation method package, has indicated that the peak field intensity of radiation in the $10 \mu \mathrm{m}$ guide may be reduced by $85 \%$ if the guide is broadened through a taper to a final width of $50 \mu \mathrm{m}$.
\end{abstract}

\section{Introduction}

Throughout the world rivers form a major source of raw water for human consumption and processes. However, many of these rivers collect both treated and untreated waste water from sewage plants and industro-agricultural processes. This polluted water is then carried through to estuarine and coastal sea areas. For instance, pesticides are in widespread use in agriculture to increase crop yields, and triazine based compounds are commonly employed as herbicides throughout the world. The persistence and mobility of the triazine herbicides is amply demonstrated by the existence of measurable quantities of atrazine and simazine in the Elbe Estuary in the North Sea ${ }^{1}$. In basins with little water exchange, such as the Mediterranean and the Baltic seas, this pollution may be the cause of serious environmental problems affecting both human health and local ecologies. Add to this the role of rivers as major sources of drinking water and it is clear that the continuous monitoring and control of water quality is vital. This monitoring should take place not only at potential sources of pollution to a river, but also along the length of the river to provide a complete picture of the state of water quality. In turn this places high demands on the cost, flexibility and ease of use of instrumentation.

Laboratory based techniques, such as high pressure liquid chromatography (HPLC) and gas chromatography with mass spectroscopy (GC-MS), exist that readily detect pollutant concentrations at the $0.1 \mu \mathrm{g} / \mathrm{l}$ level and below in water samples. However, these techniques are usually expensive and time-consuming to use due to the need for sample treatment prior to analysis. There is a need for instrumentation that will allow the rapid in-situ analysis of water samples for pollutant contamination at low cost. Immunoassay techniques offer one route towards this goal. Laboratory based enzyme-linked immunosorbent assay test kits for the 
detection of pesticides are commercially available and have been validated with natural water samples ${ }^{2}$. When coupled with an optical transducer, a lightweight, rugged, highly sensitive and potentially cheap biosensor can be developed. Several types of optical transduction mechanisms have been utilised in biosensors, although the refractometer is one of the more common. Optical biosensors based on surface plasmon resonance ${ }^{3}$, grating couplers ${ }^{4}$, Mach-Zehnder interferometers ${ }^{5}$ difference interferometers ${ }^{6}$, directional couplers ${ }^{7}$, reflectance interferometric spectroscopy ${ }^{8}$ and the resonant mirror $^{9}$ have all been reported in the literature. Fluorescence based sensors offer an attractive alternative to refractometers, with the added benefit of being less susceptible to surface interferences from complex sample media, such as humic acid bearing water. Excellent sensitivities have been reported for fluorescence based immunosensors ${ }^{10}$. In this paper we report on the development and characterisation of an intergrated optical immunofluorescence sensor for environmental monitoring. This approach allows a highly flexible sensor design process including microstructuring of the transducer to address several individual sensor positions and potentially to collect the excited fluorescence on the waveguide chip. In addition, light coupling to the waveguides by fibre pigtailing is possible making the device more suitable for use outside the laboratory environment. The waveguide surface is chemically modified to allow for specific detection of the target analyte. The sensor is characterised as a detector of the pesticide 2,4dichlorophenoxyacetic acid (2,4-D) by means of an binding inhibition immunoassay. It is regenerable and reusable for more than 50 measurement cycles. It has been shown that thin high index films deposited on the surface of the waveguide can be employed to enhance sensitivity of integrated or fibre optical interferometric and attenuation based evanescent field devices. Enhancement factors between one and two orders of magnitude have been reported $^{11,12}$. In this paper this technique is extended to the fluorescence immunoprobe reported and the enhancement of a thin high index overlayer on fluorescence excitation is demonstrated experimentally. The use of an integrated optical transducer structure leads to high optical power densities at the surface of the waveguide, and subsequently to rapid photobleaching of dye molecules bound to this surface. The rate of photobleaching of bound dye molecules has been measured as function of the power launched into the waveguide sensor without high-index films. A sensor incorporating a thin high-index film is demonstrated to cause the fastest rate of photobleaching of bound dye molecules. The use of a waveguide taper to broaden the waveguide and hence reduce the surface optical power density is demonstrated theoretically, offering the potential for greater overall sensitivity through increased signal levels as the number of bound fluorophores at the waveguide surface increases.

\section{Sensor operation and immunoassay format}

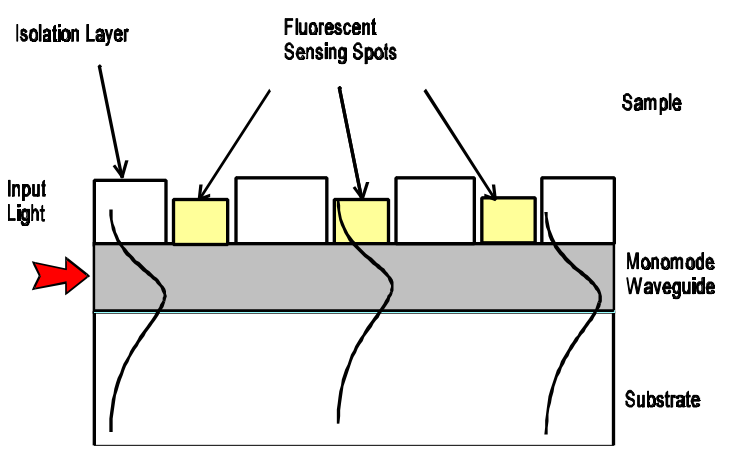

Figure 1 Schematic of the integrated optical immunofluorescence sensor.

A schematic representation of a practical immunofluorescence sensor is shown in figure 1 . The basis of the sensor is a single mode waveguide produced, for example, by thermal ion-exchange in a glass substrate. The majority of the waveguide is covered with an isolation layer, formed, for instance, by rf sputtering silica onto the waveguide surface. Small regions of the waveguide are left exposed forming interaction regions with the sample under test. In the isolated regions of the waveguide the evanescent field of the guided light traveling in the superstrate region is unable to interact with the test sample. In the exposed regions of the waveguide the evanescent field is able to interact with the sample, forming spatially separated sensing spots. If a fluorophore is brought within a few hundred nanometres of a sensing spot then the evanescent field of the guided light will excite the fluorophore, resulting in fluorescence. Incorporating a thin highindex film into the waveguide structure at the fluorescent sensing spots is a straight-forward method of enhancing device sensitivity. The highindex film will shift the optical field distribution in the waveguide towards the sensor surface, resulting 
in an increased field strength at the interface between the high-index film and the sample. In turn this increases the peak fluorescence signal excited from a given fluorophore, increasing the overall sensitivity of the sensor.

The integrated optical transducer described here has been configured to employ a binding inhibition assay, carried out in a two-step protocol, to specifically measure the concentration of 2,4-D in a water sample. The transducer surface was covalently modified to ensure optimum properties for long-term operation. A dextran layer was attached to the thiolactivated surface to reduce non-specific binding, providing a 3-dimensional matrix for immobilisation of the ligand. A 2,4-D derivative with high affinity

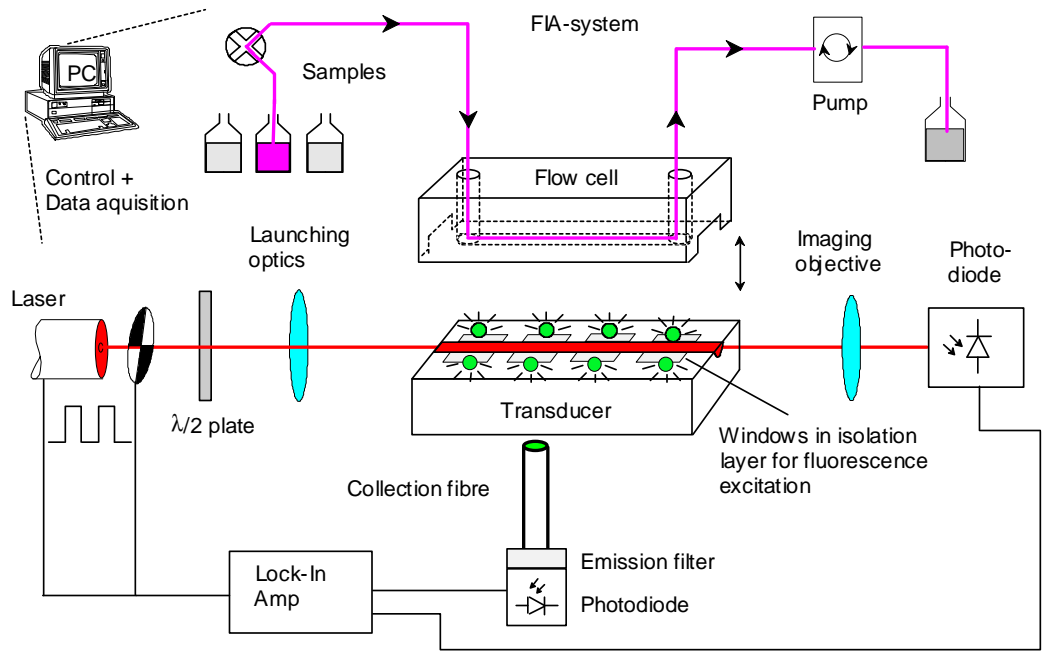

Figure 2. Experimental apparatus

to the Cy5.5 dye labeled anti-2,4-D antibodies was attached to this matrix in high concentration. These conditions ensured high binding rates and diffusioncontrolled binding at low antibody concentrations ${ }^{13}$ providing constant binding rates even after partial degradation of the immobilised ligand ${ }^{8}$. Antibodies, labeled with Cy5.5 dye, were mixed with standard 2,4-D samples and the free antibody concentration was detected by exposing the samples to the modified surface in a flow-through format. Under these conditions the total amount of antibody bound to the chemically modified sensing spots, after a fixed amount of exposure time, directly corresponds to the concentration of the free antibody, and is therefore a measure of the 2,4-D concentration in the sample. After binding is complete light at a wavelength of $633 \mathrm{~nm}$ is launched into the waveguide, exposing the dye labeled bound antibodies to the evanescent field of the guided light.
In this way fluorescence is produced from the bound dye molecules with a peak wavelength of approximately $700 \mathrm{~nm}$. The strength of the fluorescence signal is directly related to the number of excited dye molecules, and hence the number of bound antibodies, and ultimately to the concentration of 2,4-D in the test sample.

\section{Experimental}

\subsection{Immunoassays}

For this characterisation the waveguide sensor consisted of potassium ion-exchanged waveguides fabricated in a Pyrex substrate. An ion-exchange time of 8 hours, at a temperature of $395{ }^{\circ} \mathrm{C}$, was used to produce single mode waveguides at $633 \mathrm{~nm}$. The sensor did not utilise an isolation layer as a suitable low refractive index material had not been identified. Surface modification of the optical sensor chip, to allow the specific detection of 2,4-D, was carried out according to established procedures ${ }^{13}$.

The experimental apparatus is shown schematically in figure 2. Fluorescence was excited at $633 \mathrm{~nm}$ by a mechanically

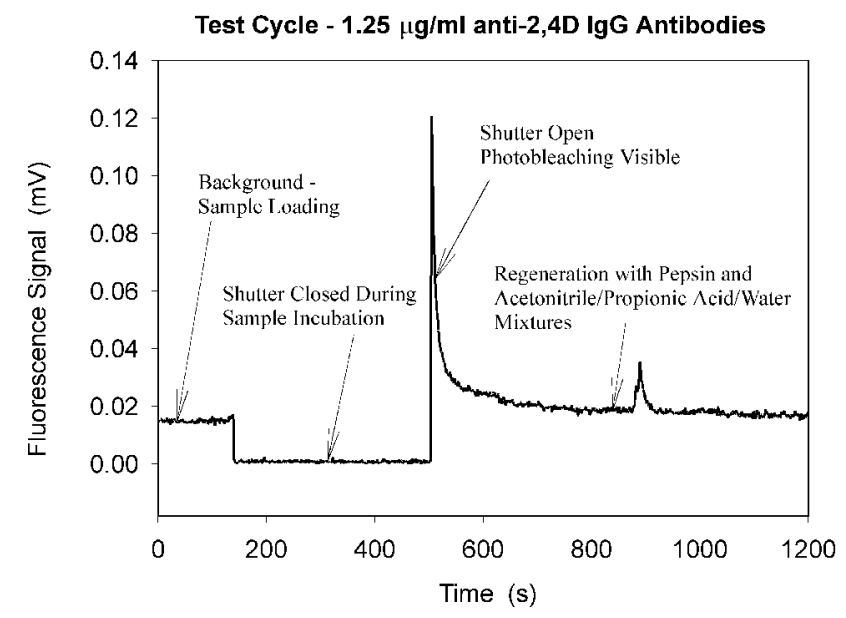

Figure 3. Sensor test-cycle using a solution of $1.25 \mu \mathrm{g} / \mathrm{ml}$ of anti-2,4D antibodies 


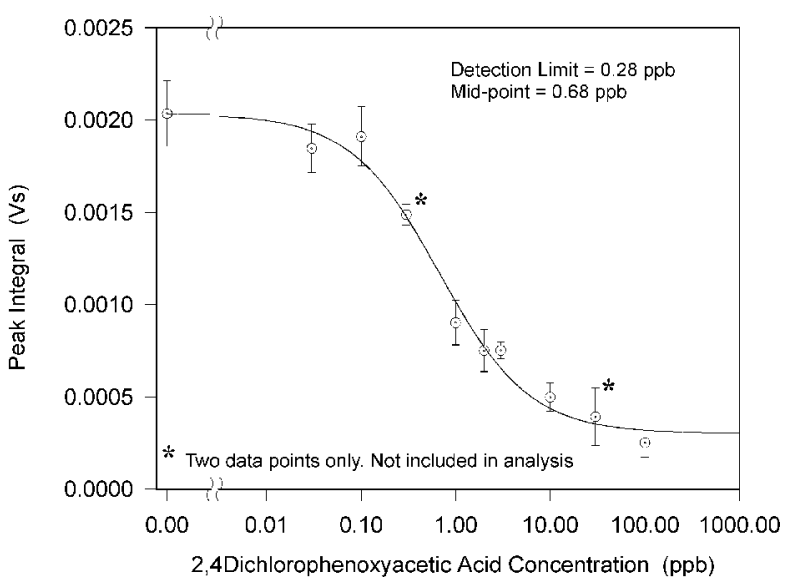

Figure 4. Averaged calibration curve for 2,4-D

chopped He-Ne laser, end-fire coupled into the test waveguide. The fluorescence was collected by a high numerical aperture polymer optical fibre located under the sensor chip, filtered to remove stray pump light, and detected by a silicon photodiode. The diode signal was acquired by a lock-in amplifier using phase sensitive detection and recorded by a PC. Control of whether the antibodies, and hence dye molecules, bound at the sensor surface were exposed to the He-Ne radiation was achieved through the use of a computer controlled electro-mechanical shutter in the laser beam path. A test-cycle using a Cy5.5 labeled anti-2,4-D antibody concentration of 1.25 $\mu \mathrm{g} / \mathrm{ml}$ is plotted in figure 3 . Each antibody molecule had been tagged with an average of 2.3 dye molecules. The plot has been labeled with the various components of the test-cycle showing, sample loading and incubation, fluorescence measurement and surface regeneration. The average background signal was calculated to be $0.015 \mathrm{mV}$ and a peak signal of $0.12 \mathrm{mV}$ was recorded. Sensitivity of the pre-amplified silicon photodiode was $48 \mu \mathrm{V} / \mathrm{pW}$. After scaling the measured signal by a factor of 2.2, to account for the lock-in amplifier measuring only the first harmonic fraction of the signal, the background and peak signal correspond to optical powers of 0.7 and $5.5 \mathrm{pW}$ respectively.

Three calibration curves were produced using this concentration of $1.25 \mu \mathrm{g} / \mathrm{ml}$ of dye-labeled antibodies, and a range of 2,4-D standards in phosphate buffered saline covering the concentration range of $0-100 \mathrm{ppb}$. The signal was evaluated as the difference between the fluorescence signal integrated over $100 \mathrm{~s}$ after binding and the background signal similarly integrated before binding. After each assay the transducer surface was regenerated for immediate reuse. The average of the three curves is shown in figure 4 . The averaged data set has been fitted by a sigmoidal curve, representing a close approximation to the actual shape of the calibration curve. The detection limit for 2,4-D was calculated as $0.28 \mathrm{ppb}$, with a mid-point of the response at $0.68 \mathrm{ppb}$. This detection limit was taken to be a point on the fitted curve of figure 4 which was three standard deviations of the mean below the average of the blank value.

A separate waveguide chip, also fabricated by potassium ion-exchange in Pyrex, and coated with a $20 \mathrm{~nm}$ film of tantalum pentoxide $(\mathrm{n}=2.1)$ was surface modified to allow the detection of 2,4-D as above. The modification employed was the same as for a silica surface; however, this speculative modification allowed a demonstration of the sensitivity enhancement that may be expected from using thin high-index films in the sensor structure. A test-cycle was recorded using this sensor and a blank sample containing no $2,4-\mathrm{D}$, but $1.25 \mu \mathrm{g} / \mathrm{ml}$ of anti2,4-D antibodies. The peak fluorescence signal measured in the absence of 2,4-D was, after background subtraction, $0.71 \mathrm{mV}$. This is greater than 6 times that achieved with the same antibody concentration and a modified sensor without a highindex film.

\subsection{Photobleaching}

The rate of photobleaching of the dye molecules is

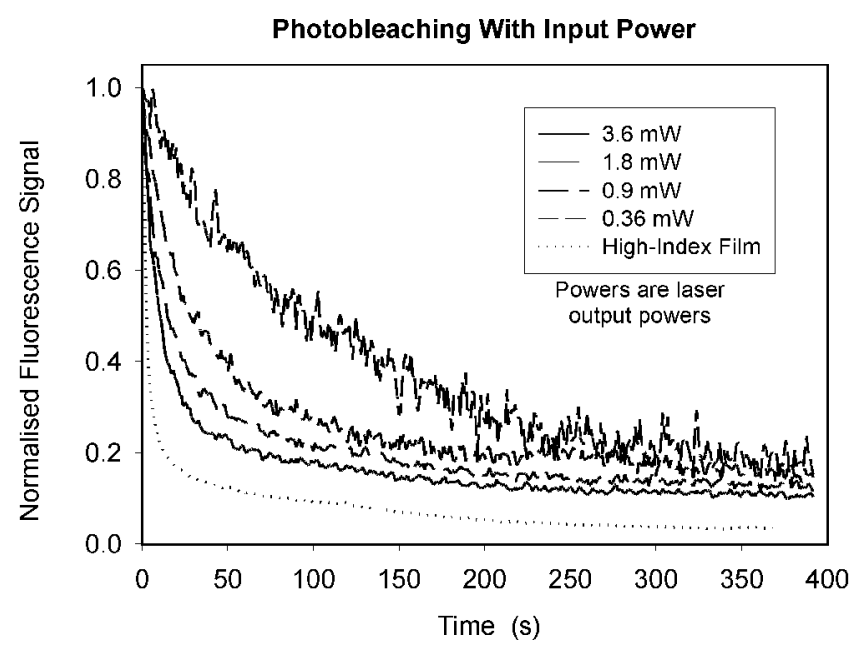

Figure 5. Variation in rate of photobleaching of excited Cy5.5 dye molecules as a function of laser output power. 
dependent on the power density of the radiation at the sensor surface where the dye molecules are bound. The integrated optical sensor makes use of an active area of $0.02 \mathrm{~mm}^{2}$ over which the bound, dye-labeled antibodies, are excited by the light guided in the ion-exchanged waveguide. This area is bounded by the width of the waveguide, approximately $10 \mu \mathrm{m}$, and the length of each sensing spot, chosen to be $2 \mathrm{~mm}$. This is expected to cause rapid photobleaching of the bound dye molecules at the sensor surface.
Making use of the sensor characterised as a detector for 2,4-D several test-cycles were measured using 2.5 $\mu \mathrm{g} / \mathrm{ml}$ of anti-2,4-D antibodies. For each test-cycle the output power of the laser was reduced so that the variation in the rate of photobleaching of the dye molecules as a function of the power density at the sensor surface could be monitored. Figure 5 plots the decay in the fluorescent signal with time after binding and opening of the shutter. The data recorded for the high-index film was taken at a later date and the relation of the absolute power density to that of the first experiment is not known, although it is expected to be higher for the high-index film measurement.

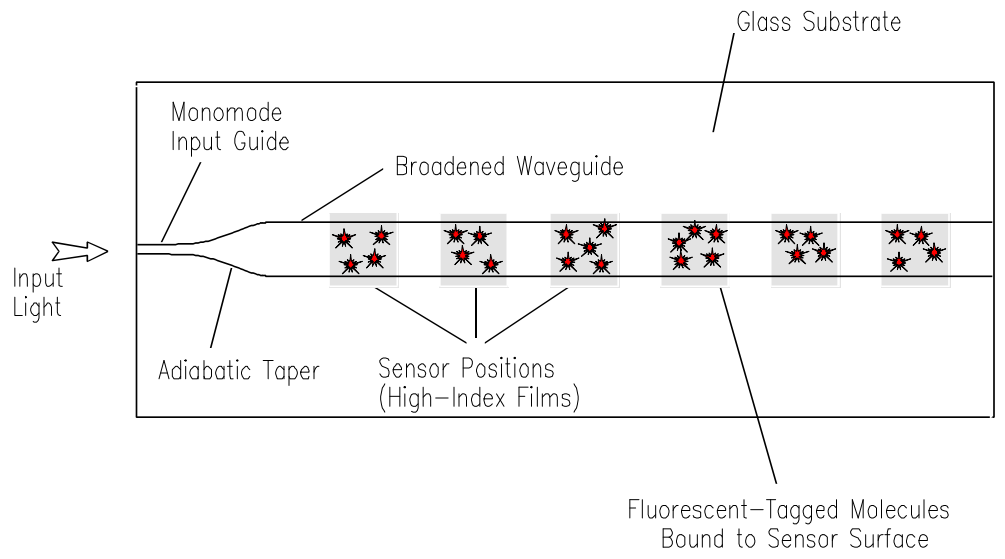

Figure 6 Schematic of broadened waveguide structure for reducing photobleaching.
For each curve a single exponential was fitted and the time constant determined. The calculated time constants are listed in table 1. However, at laser output powers greater than $0.36 \mathrm{~mW}$, and in the case of the high-index film, a single exponential fit does not appear to be the best choice of curve. As a comparison, each data set has also been fitted by a double exponential curve, which appears to fit the data better for all laser output powers greater than $0.36 \mathrm{~mW}$. The time constants of the two exponentials in the double exponential fit are also listed in table 1.

\begin{tabular}{|c|c|c|c|}
\hline $\begin{array}{c}\text { Laser Output } \\
\text { Power (mW) }\end{array}$ & $\begin{array}{c}\text { Single Exponential } \\
\text { Time Constant (s) }\end{array}$ & $\begin{array}{c}\text { Double Exponential } \\
\text { First Time Constant (s) }\end{array}$ & $\begin{array}{c}\text { Double Exponential } \\
\text { Second Time Constant } \\
(\mathrm{s})\end{array}$ \\
\hline 3.6 & 18.3 & 8.9 & 97.0 \\
\hline 1.8 & 30.3 & 11.1 & 94.3 \\
\hline 0.9 & 41.4 & 22.0 & 119.0 \\
\hline 0.36 & 110.9 & 119.8 & 13.28 \\
\hline High-Index Film & 3.2 & 5.6 & 108.0 \\
\hline
\end{tabular}

Table 1 Time constants of the single and double exponential curves fitted to the experimental measurements of figure 5.

The physical significance of this change of form of the experimentally measured data is not clear at the present time. It does not have a significant impact on the operation of the device as a biochemical sensor. Reduction of photobleaching, without a reduction in the peak excited fluorescence power, leading to greater sensitivities is a development aim for this immunofluorescence sensor. A potential mechanism to achieve this aim will be to increase the waveguide width, maintaining the same level of 


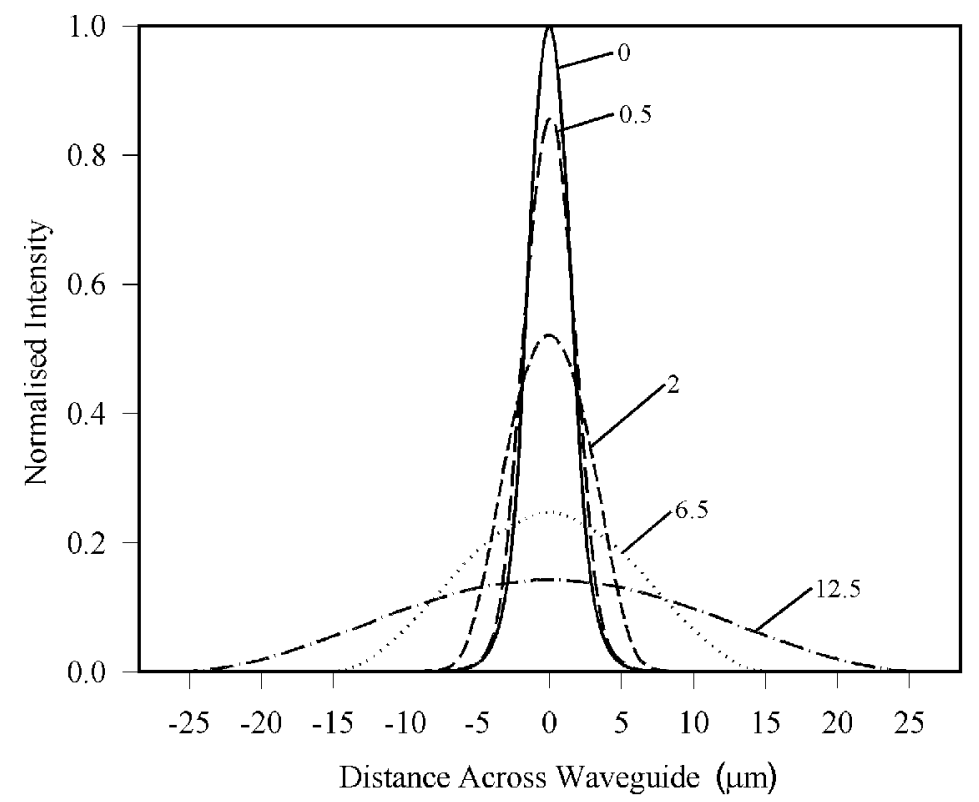

Figure 7 Broadening of the TE modal fields on passing through a $12.5 \mathrm{~mm}$ linear taper with a final width of $50 \mu \mathrm{m}$. Curves are labelled with distance along the taper in $\mathbf{~ m m}$

power launched into the waveguide. This will lead to a reduction in the power density of radiation experienced by each fluorophore, balanced by an proportional increase in the number of excited fluorophores. Such a change will leave the total

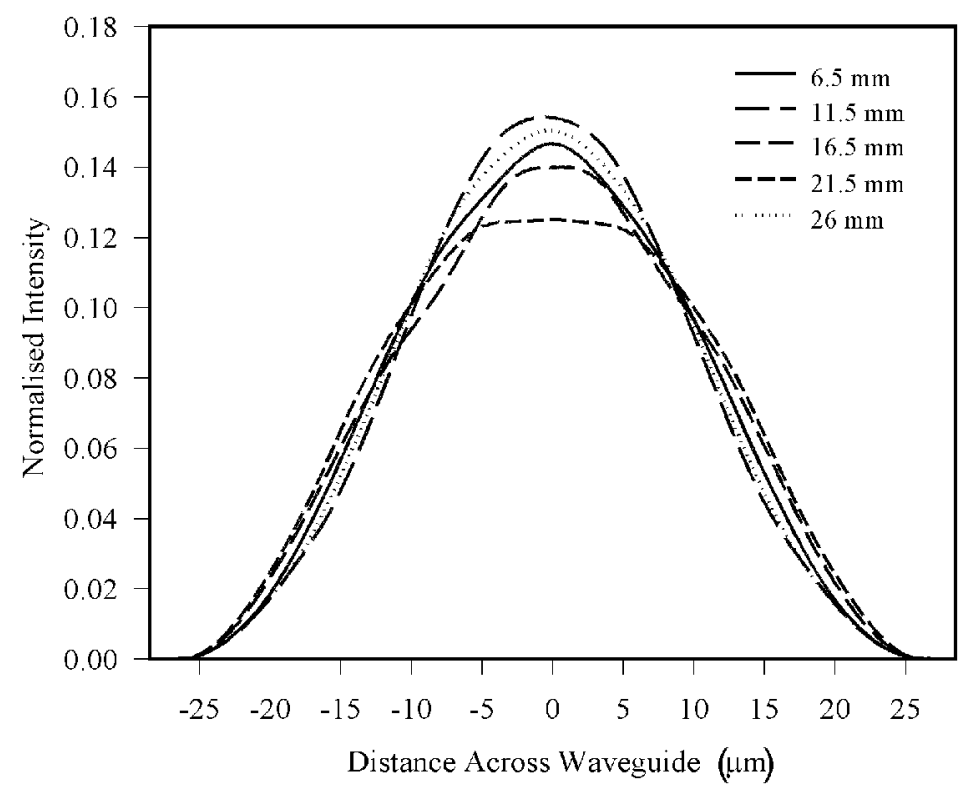

Figure 8 Modal field profiles in a $50 \mu \mathrm{m}$ wide waveguide after excitation through a $12.5 \mathrm{~mm}$ long linear taper. Curves are labelled with distance along the waveguide in $\mathrm{mm}$. amount of emitted power the same and reduce the rate of photobleaching of the exposed fluorophores.

\section{Reducing the rate of photobleaching}

The rate of photobleaching of the bound fluorophores will be reduced if the power density of the excitation radiation at the surface of the waveguide is reduced. Decreasing the rate of photobleaching offers two distinct advantages. First, data acquisition becomes more straightforward as the fluorescence signal changes less rapidly than previously. Second, a wider waveguide increases the number of bound fluorophores excited during the measurement leading to a greater measured signal. This second advantage is expected to lead to an increased signal to noise ratio, and hence an improved detection limit for the sensor.

In order to maintain a uniform power distribution in a wide, and hence highly multi-moded, waveguide it is desirable to excite only the fundamental mode of the waveguide. Figure 6 shows a schematic sensor structure offering the potential to meet this aim. A monomode waveguide is excited from the laser source. This monomode waveguide is then broadened very slowly, by a taper in the waveguide walls, to the required width. If the rate of increase in the width of the waveguide is slow enough the broadening of the modal field is essentially adiabatic resulting in all of the launched light remaining in the fundamental mode of the waveguide. A theoretical study of linear tapers of different lengths and final waveguide widths has been made using a commercial Beam Propagation Method (BPM) computer program. A transition length of $12.5 \mathrm{~mm}$ was studied for use in waveguide devices $50 \mathrm{~mm}$ long. The device length is limited by the availability of cheap soda-lime or Pyrex glass substrates, 
and the length of the transition by the need to incorporate 6 sensing positions along each waveguide. The waveguide parameters were chosen to match those of a potassium ion-exchanged waveguide in a soda-lime glass substrate, excited at $633 \mathrm{~nm}$. The waveguide is coated with a silica film, acting as an isolation layer. Final broadened waveguide widths of 50 to $150 \mu \mathrm{m}$ were studied.

Figure 7 plots the changing field profile of light passing through a $12.5 \mathrm{~mm}$ long linear taper where the waveguide width changes from an initial $4 \mu \mathrm{m}$ to a final value of $50 \mu \mathrm{m}$. The peak intensity at the end of the taper has fallen to $14 \%$ of the value at the narrow end. Transmission of light through the taper is greater than $99 \%$, indicating that very little light is lost to scattering as the waveguide shape changes along the tapered region. Figure 8 plots the field profile of the light guided along the broadened section of the waveguide, with a fixed width of $50 \mu \mathrm{m}$. To accommodate six sensor positions, each $2 \mathrm{~mm}$ long and separated by $2 \mathrm{~mm}$, the broadened waveguide section has a length of $26 \mathrm{~mm}$. The field profile along the waveguide is not uniform, as would be expected if only the fundamental mode of the centre of the waveguide is relatively small, with the minimum being $75 \%$ of the maximum value.

For a waveguide with a width of $150 \mu \mathrm{m}$, also excited by a $12.5 \mathrm{~mm}$ long taper with a starting width of $4 \mu \mathrm{m}$, the excitation of modes other than the fundamental has increased further. The field profile plot of figure 9 displays a significant amount of modal beating along the waveguide. In turn this will create different rates of photobleaching of bound dye molecules not only along the waveguide length, but also across it.

Due to the physical constraints on the allowable length of the tapered region in a practical device a truly adiabatic transition cannot be achieved, as can be seen from figures 8 and 9 . One possibility to make the transition closer to adiabatic is to use a parabolic wall shape causing the transition to be slowest where there is the greatest number of modes, thus reducing the excitation of higher order modes.

\section{Conclusions}

An integrated optical immunofluorescence sensor has been characterised as a sensor for the pesticide 2,4dichlorophenoxyacetic acid. A calculated detection limit of $0.28 \mu \mathrm{g} / \mathrm{l}$ was achieved, with the mid-point of the calibration curve occurring at $0.68 \mu \mathrm{g} / \mathrm{l}$. The effect of a $20 \mathrm{~nm}$ thick tantalum pentoxide film deposited on the waveguide surface to enhance device sensitivity has been studied. A six fold increase in the peak fluorescence signal was observed in antibody binding experiments in comparison with the uncoated device. The integrated optical sensor utilises an active area of $0.02 \mathrm{~mm}^{2}$ for each sensing spot, and may incorporate up to six spatially separated sensing spots along a single waveguide. These multiple spots allow the possibility of with distance along the waveguide in $\mathbf{m m}$.

waveguide was excited. This variation in field profile is a result of the modal beating along the structure. The variation in field intensity along the detecting an equal number of target analytes in a single test. 
Fast rates of photobleaching were observed, especially for the sensor incorporating the thin tantalum pentoxide film. A strategy to reduce the rate of photobleaching, by broadening the waveguide through a linear taper, has been identified and theoretically studied. The modelling of tapered waveguide structures, using a commercial beam propagation method package, has indicated that the peak field intensity of radiation in a $10 \mu \mathrm{m}$ guide may be reduced by $85 \%$ if the guide is broadened through a $12.5 \mathrm{~mm}$ taper to a final width of $50 \mu \mathrm{m}$. Lower rates of photobleaching will result in a greater signal to noise ratio for the sensor, leading to greater overall device sensitivity. Combining the concept of a broadened waveguide to reduce the rate of photobleaching with the existing sensor structure will give rise to a highly sensitive integrated optical immunofluorescence sensor capable of multi-analyte detection.

\section{Acknowledgments}

The authors gratefully acknowledge the financial support of the EU Environment and Climate Programme, contract number ENV4-CT95-0066. The Optoelectronics Research Centre is an Interdisciplinary Research Centre supported by the UK Engineering and Physical Sciences Research Council.

\section{References}

1. Bester, K. \& Hühnerfuss, H. (1993). Triazines in the Baltic and North Sea. Marine Pollution Bulletin, 26, 423-427.

2. Mouvet, C., Broussard, S., Jeannot, R., Maciag, C., Abuknesha, R. \& Ismail, G. (1995). Validation of commercially available ELISA microtiter plates for triazines in water samples. Analytica Chimica Acta, 311, 331-339.

3. Mouvet, C., Harris, R. D., Maciag, C., Luff, B. J., Wilkinson, J. S., Piehler, J., Brecht, A., Gauglitz, G., \& Abuknesha, R. A. (1997). Determination of Simazine in Water Samples by Waveguide Surface Plasmon Resonance. Analytica Chimica Acta, 338, 109-117.
4. Lukosz, W., Clerc, D., Nellen, Ph. M., Stamm, Ch. \& Weiss, P. (1991). Output grating couplers on planar optical waveguides as direct immunosensors. Biosensors and Bioelectronics, 6, 227-232.

5. Heideman, R. G., Kooyman, R. P. H. \& Greve, J. (1993). Performance of a highly sensitive optical waveguide Mach-Zehnder interferometer immunosensor. Sensors and Actuators B, 10, 209217.

6. Fattinger, Ch., Koller, H., Schlatter, D. \& Wehrli, P. (1993). The difference interferometer: a highly sensitive optical probe for quantification of molecular surface concentration. Biosensors and Bioelectronics, 8, 99-107.

7. Luff, B. J., Harris, R. D., Wilkinson, J. S., Wilson, R. \& Schiffrin, D. J. (1996a). Integrated optical directional coupler biosensor. Optics Letters, 21, 618-620.

8. Brecht, A., Piehler, J., Lang, G. \& Gauglitz, G. (1995). A direct optical immunosensor for atrazine detection. Analytica Chimica Acta, 311, 289-299.

9. Goddard, N. J., Pollard-Knight, D. \& Maule, C. H. (1994). Real-time Biomolecular Interaction Analysis Using the Resonant Mirror Sensor. Analyst, 119, 583-588.

10. Plowman T.E., Reichert W.M., Peters C.R., Wang H.K., Christensen D.A., Heron J.N. (1996). Femtomolar sensitvity using a channel-etched thin film waveguide fluoroimmunosensor, Bioesens. \& Bioelectron., 11, 149-160.

11. Muhammad F.A., Stewart G. (1992). Sensitivity Enhancement of D-fibre and application for chemical sensing, Int. J. Optoelectron., 7(6), 705-721.

12. Quigley G.R., Harris R.D., Wilkinson J.S.. Sensitivity enhancement of integrated optical sensors by thin high index films. Proc. 8th European Conference on Integrated Optics (ECIO), Stockholm, Sweden, April 2 - April 4 (1997), 326329.

13. Piehler, J., Brecht, A., Geckeler, K. E. \& Gauglitz, G. (1996). Surface modification for direct immunoprobes. Biosensors and Bioelectronics, 11, 579-590. 